\title{
Microcontroller Based Model Design of a Train Collision Avoidance System
}

\author{
Elisha C Mabunda ${ }^{1}$ Cleophas D K Mutepfe ${ }^{2}$ \\ Elisha C Mabunda ${ }^{1}$ (+263 773214 757),Cleophas D K Mutepfe ${ }^{2}(+263775$ 272278) \\ 1 and 2 Department of Electrical Engineering University of Zimbabwe P.O. Box MP167 Mt Pleasant, Harare
}

\begin{abstract}
This article introduces a design concept to curb the rail accidents such as collisions and derailment as obtaining in theSouthern African Region. The design comprises apparatus for detecting the presence of an approaching locomotive within a specified limited area, while being insensitive to the presence of objects outside the specified region and triggering braking circuits which will stop the train. The system is integrated with the braking system of the train and is capable of detecting the possibility of collisions in real time. The design is intended to automate fully the communication system between locomotives so that rail traffic accidents can be minimized or eliminated.Forauthentication purposes the design incorporates addresses of encoder and decoder that are matched, this way the detection unit will never respond to stray signals or other signals within the spectrum. The main advantage of this electronically controlled braking system would be reduced braking distance and increased railway traffic under safe conditions. This paper presents a solution that uses a microcontroller to execute a predetermined software algorithm for precise braking of the train.
\end{abstract}

Keywords: Acceleration, Braking, Communication, collision avoidance Modulation

\section{INTRODUCTION}

Communication is a key issue in traffic management and tracking and is not an exception in railroad traffic control. With most of the communication and signaling equipment in Southern African Region are now obsolete and dilapidated, accidents are prone to occur which in most cases are fatal claiming a lot of life. Rail accidents are inevitable where communication is inefficient hence the need to device systems which will enable trains to communicate automatically, if we are to harness these problems. Currently, organizations like NRZ are implementing the radio communication system where the train driver would get information about the rail status from a control officer at a nearby station. The status is reflected on the point circuit, where points are track switches which are completed when a train passes a certain section. As the train progresses in its track, the driver is continuously reminded to check his/her points and to drive with caution when approaching points whose status cannot be clearly determined. In case the radio communication system fails, there is no collision avoidance measure being implemented to curb these tragedies; hence this project would greatly improve safety in rail traffic.

\subsection{Breaking Distance}

\section{BRAKING SYSTEM}

The dynamics of a train moving with speed $v$ along a track inclined at an angle $\alpha$ to the horizontal aredetermined by the forces shown in Fig 1 below.

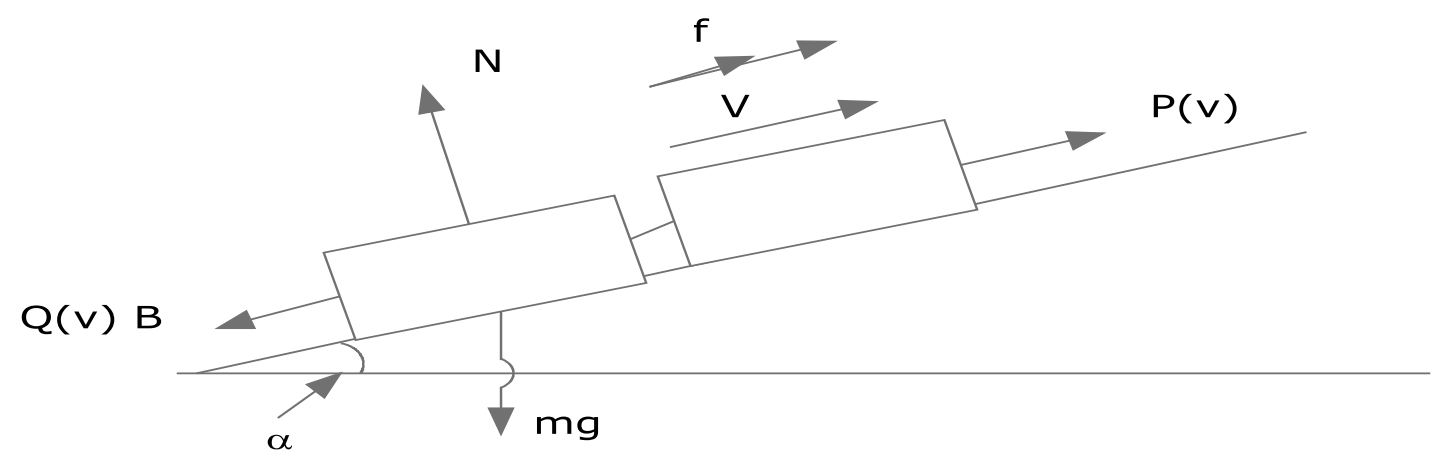

Fig 1. Diagram showing the dynamics of the train 
Where:

$P(v)$ is the tractive effort of the locomotive;

$Q(v)$ is the drag;

$B \quad$ is the brake force;

$m g \quad$ is the weight of the train;

$N \quad$ is the reaction of the track.

By Newton's second law of motion [1], the acceleration, $f$ is given by:

$m f=P(v)-Q(v)-B-m g \sin \alpha$

This equation can be used to derive a number of relationships that are important to different aspects of railway operation.

The brake force available depends on two factors:

1. the adhesion between the rail and the wheels being braked, and

2. the normal reaction of the rail on the wheels being braked (and hence on the weight per braked wheel)

Generally, it is specified as a fraction ( $\beta$, say) of the total weight of the train:

$B=m g \beta$

A typical value for $\beta$ is 0.09

A train reaches its maximum speed when available tractive effort just balances the sum of drag and downhill gravitational force, reducing the acceleration to zero. Consequently, the maximum speed is found by solving:

$P(v)-Q(v)-m g \gamma=0$

where $\gamma \equiv \sin \alpha$ is the gradient.

Since the approximation to $P(v)$ is linear within each segment, and that for $Q(v)$ is quadratic, the calculation of maximum speed for a particular gradient reduces to the solution of a quadratic equation. However, in order to determine which segment of the tractive effort approximation should be used for a given gradient, it is useful first to establish a set of gradient values $\left\{\gamma_{i}\right\}$ whose corresponding maximum speeds are equal to the transition speeds $v_{i}$ between segments.

To calculate how long it will take for a train to come to rest when the locomotive power is cut off and the brakes are applied, and how far it will travel in this time, set $P(v)=0$. Since acceleration, $\mathrm{f}$ is rate of change of velocity, a differential equation:

$m \frac{d v}{d t}=-B-Q(v)-m g \gamma$

Describes the motion and once the initial speed is given, defines $v$ as a function of time $t$.

Since the braking force $B$ is essentially a constant, independent of speed, the differential equation can be integrated by separation of variables[2] leading to:

$\int_{V}^{0} \frac{m d v}{m g(\beta+\gamma)+Q(v)}=-\int_{0}^{T} d t$

The drag $Q(v)$ is approximated by a quadratic function of speed:

$$
Q(v)=q_{0}+q_{1} v+q_{2} v^{2}
$$

it becomes clear that the braking time $T$ required from speed $v$ is obtained as the integral:

$$
T(v)=\int_{0}^{v} \frac{d u}{a u^{2}+b u+c}
$$

Where:

$$
a=q_{2} / m ; b=q_{1} / m ; c=q_{0} / m+g(\beta+\gamma) .
$$

From this result, a further integration is needed to recover the distance travelled as a function of time. A simpler alternative is to calculate the braking distance directly by writing:

$f=\frac{d v}{d t}=\frac{d v}{d s} \frac{d s}{d t}=v \frac{d v}{d s}$

in the original equation, to give:

$$
m v \frac{d v}{d s}=-B-Q(v)-m g \gamma
$$


This is a relation between distance $\mathrm{s}$ and speed $\mathrm{v}$.

This differential equation can also be integrated by separation of variables, leading to:

$\int_{V}^{0} \frac{m v d v}{m g(\beta+\gamma)+Q(v)}=-\int_{0}^{S} d s$

And hence the braking distance $S$ required from speed $v$ is obtained as the integral:

$S(v)=\int_{0}^{v} \frac{u d u}{a u^{2}+b u+c}$

Where again

$$
a=q_{2} / m ; b=q_{1} / m ; c=q_{0} / m+g(\beta+\gamma) .
$$

\subsection{Time spent accelerating to required speed}

Each stop that a train makes during its journey involves three phases: braking to a standstill, remaining stationary to set down and pick up passengers, and accelerating to the required line speed. An appropriate allowance for the time taken for each of these phases, as well as other braking and acceleration manoeuvres (e.g. to traverse a set of points) must be included when drawing up realistic timetables. Section 2.1 considered time taken for braking; calculation of the time taken in acceleration is similar, but somewhat more involved because of the piecewise-linear approximation to the variation of tractive effort with speed.

Setting $B=0$ produces the differential equation:

$m \frac{d v}{d t}=P(v)-Q(v)-m g \gamma$

which, once the initial speed is given, defines $v$ as a function of time $t$.

Since the tractive effort $P(v)$ is a function of speed only, the differential equation can be integrated by separation of variables[3], leading to:

$\int_{0}^{V} \frac{m d v}{P(v)-Q(v)-m g \gamma}=\int_{0}^{T} d t$.

Because the approximation to $P(v)$ is a piecewise-linear function of speed, and the drag $Q(v)$ is approximated by a quadratic function of speed, the time $T$ required to accelerate to speed $v$ can be obtained by splitting the motion into segments. A transition between segments is required when the speed reaches one of the breakpoint speeds in the piecewise-linear approximation for $P(v)$.

For each segment, the elapsed time and the distance travelled can be expressed as:

$$
T(v)=\int_{v_{s}}^{v_{f}} \frac{d u}{a u^{2}+b u+c} S(v)=\int_{v_{s}}^{v_{f}} \frac{u d u}{a u^{2}+b u+c}
$$

Where $v_{s}$ and $v_{f}$ are, respectively, starting and finishing speeds for the segment, and the parameters:

$$
a=-q_{2} / m \quad ; \quad b=\left(p_{1}-q_{1}\right) / m \quad ; \quad c=\left(p_{0}-q_{0}\right) / m-g \gamma .
$$

all remain constant throughout the segment. The total time or distance needed to accelerate to a given speed is found by summing over the segments.

3. System Implementation

The implementation of this project can be explained using the diagram below

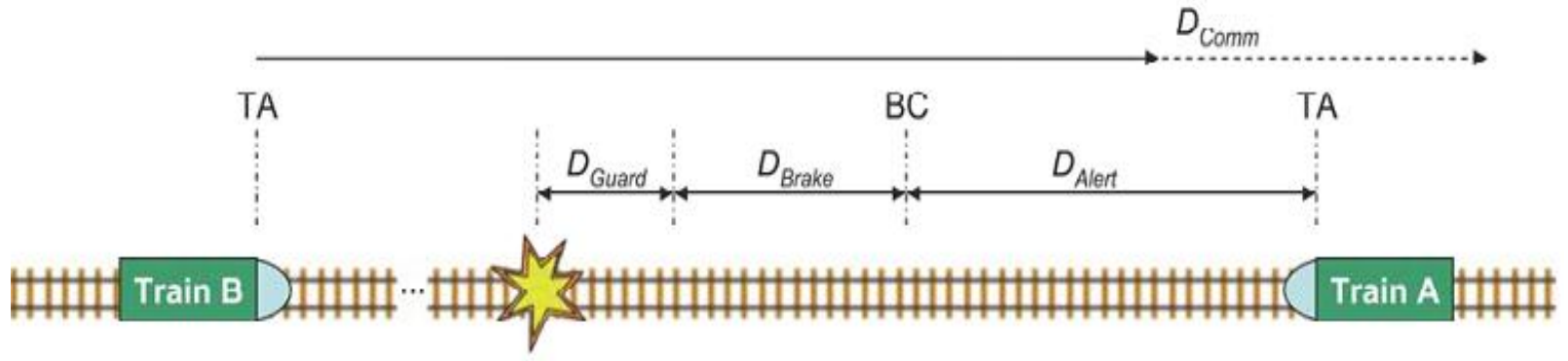

Fig.2 Diagram showing Guard distance. 
Train A in Fig. 2 will send a coded infrared signal which is received by a receiver unit mounted on train B in its proximity, with a matching address. These pulses are then passed through the decoder and if code is recognized, it sets output port high. The output is then connected to the braking system directly or via a microprocessor. Train B will also send a reciprocal signal to confirm that it has received a signal and that braking is in progress. Train B upon reception of this signal will also start applying brakes.

Because of the long braking distances due to current state of the railway lines in Southern Africa, an alert and advisory (or command) concept, promises a major safety gain. In the first step a Traffic Alert (TA) signal shall warn the train driver in case of a detected close approach to another Railway Collision Avoidance System (RCAS) unit on a collision course. TA will be activated with a fixed time prior to the time when the train has to start braking, given that it proceeds with the momentary speed. Thus, the train driver is prepared to receive a Braking Command (BC) in a second step, which is signaled after Alert $t$ seconds in order to avoid the collision. To allow for a safe braking, a guard distance is added to the braking distance to assure that the trains come to a full stop under all environmental conditions for example in rainy conditions a train would take long to stop due to reduced friction[4]. The real braking distance might vary due to the fact that adhesion and friction between wheels and rails are changing depending on the environmental conditions, which are difficult to be quantified. In order to minimize false alarms in regular operation conditions and to efficiently use the track infrastructure, the braking distance shall not be overestimated. On the other hand, an underestimation of the braking distance would cause collisions. Therefore we propose to add a guard distance Guard D as depicted in Fig. 2 above. Under the assumption that all involved vehicles are aware of the critical situation it means we can guarantee that the communication range is larger than the sum of the total braking distances.

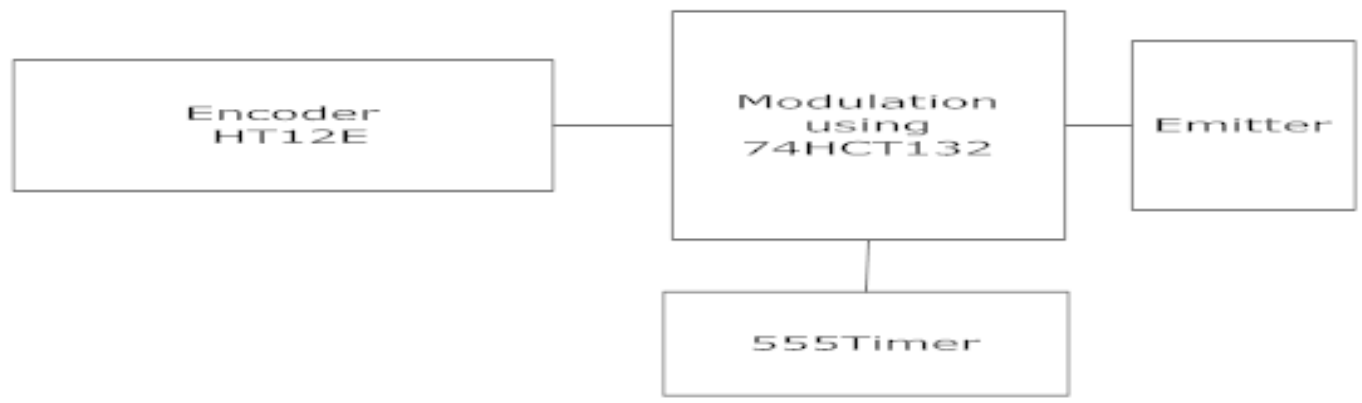

Fig.3 Transmitter Block diagram

The serial communication system will employ a pair of infrared transceivers with one mounted at one of the locomotive and another transceiver mounted at the opposite end of a remote railway train in alignment with each other. Both the receiver and transmitter are coupled to the controller (microprocessor control circuit) and to power source.

The controller is further coupled to the braking system of the train and is capable of controlling operation thereof. In the simulated laboratory version the dc motor operating the test vehicle resemble the braking system and it is controlled by a relayThe receiver circuit is illustrated inFig.4.

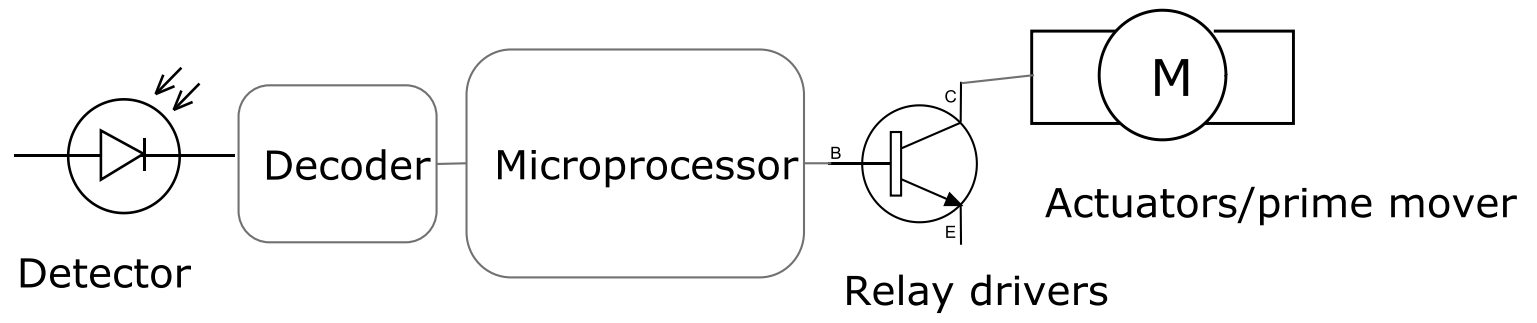

Fig. 4 Receiver Block diagram

\section{IDENTIFICATION}

In order to differentiate between the desired signal and infrared emissions from other sources such as the sun, each transmitted signal should be coded. The encoder and decoder share the same address such that when a signal is received decoder checks if it was sent from the oncoming locomotive or not. If a match exists, the decoder indicates this by setting its output to a high voltage. To achieve this HT-12E encoder and corresponding HT-12D decoder are used. These are CMOS integrated circuits that are manufactured by Holtek for remote control applications such as: car alarm systems, cordless phones, security systems and such others $[5]$. 


\subsection{Transmitter}

Fig 4 shows the details of the transmitting circuit comprising of a Schmitt trigger and an IR-LED driver. Pins 1 and 2 are inputs to the Schmitt trigger and are derived from the 555 timer and the microcontroller.

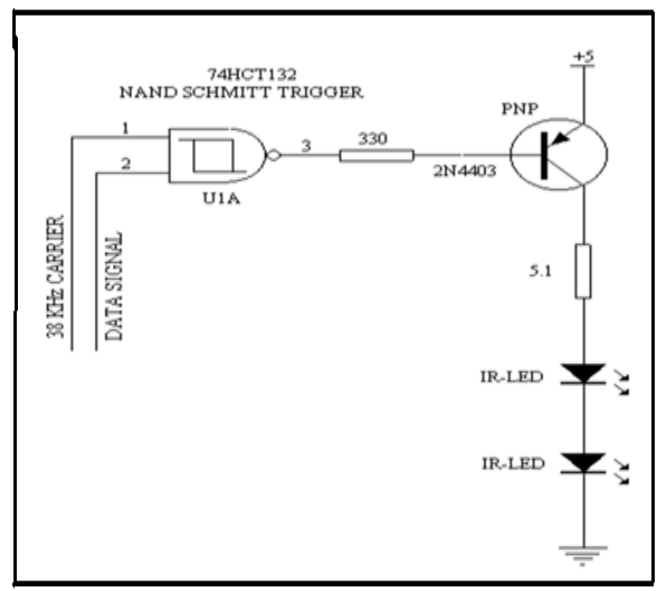

Fig. 5 Transmitter circuit diagram

IV.

THE CARRIER SIGNAL

In this project a $38 \mathrm{kHz}$ carrier signal is generated using the 555 timer. The timer is connected as an astable multi-vibrator. An a-stable circuit produces a square wave, which is a digital waveform with sharp transitions between low $(0 \mathrm{~V})$ and high $(+\mathrm{Vs})$. This signal is then modulated using the modulating signal from the HT-12E encoder.

In the time delay mode of operation, the time is precisely controlled by one external high stability resistor and capacitor. For a stable operation as an oscillator, the free running frequency and the duty cycle are both accurately controlled with two external resistors and one capacitor. The circuit may be triggered and reset on falling waveforms, and the output structure can source or sink up to $200 \mathrm{~mA}$.

\subsection{Determination of Duty Cycle}

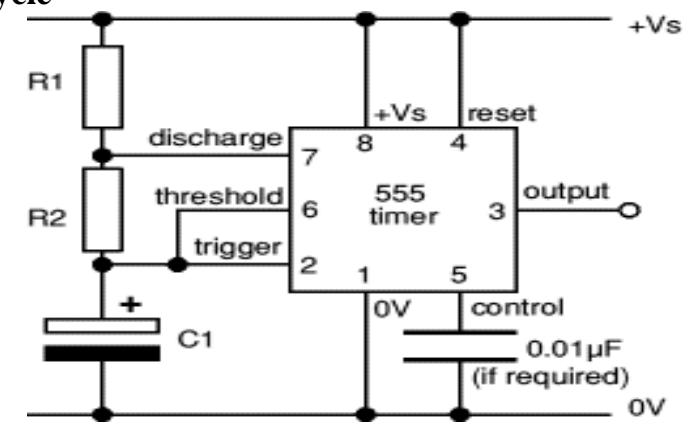

Fig. 6 Configuration of 555 timer to obtain the required duty cycle

The time period can be split into two parts: $\boldsymbol{T}=\boldsymbol{T} \boldsymbol{m}+\boldsymbol{T} \boldsymbol{s}$

Mark time (output high): $\mathrm{Tm}=0.7 \times C 1 \times(R 1+R 2)$

Space time (output low): $T s=0.7 \times R 2 \times C 1$

Many circuits require Tm and Ts to be almost equal that is $50 \%$ duty cycle. This is achieved by making R2 much larger than R1. [6]

\subsection{Choosing R1, R2 and $\mathrm{C} 1$}

According to specifications $\mathrm{R} 1$ and $\mathrm{R} 2$ should be in the range $1 \mathrm{k} \Omega$ to $1 \mathrm{M} \Omega$. It is best to choose $\mathrm{C} 1$ first because capacitors are available in just a few values.

Choose $\mathrm{C} 1$ to suit the frequency range required and $\mathrm{R} 2$ for the specific frequency. $\mathrm{R} 1$ is chosen to be about a tenth of $\mathrm{R} 2(1 \mathrm{k} \Omega \mathrm{min}$.)

$R 2=\frac{0.7}{f \times C 1}$

Signal diodes used were the $1 \mathrm{~N} 914$ types. This circuit is best used at Vcc $=15 \mathrm{~V}$. 


\subsubsection{Considerations with using a 555 timer}

Correct supply filtering and bypassing is critical in most operations, a capacitor between $0.01 \mu \mathrm{F}$ to $10 \mu \mathrm{F}$, depending upon the application should be placed as close as possible to the 555 timer supply pin. Owing to internal design considerations the 555 timer can generate large current spikes on the supply line. Care should be taken in selecting stable resistors and capacitors for timing components in the 555 timer. Capacitors must be low leakage types with very low dielectric absorption properties. Electrolytic and Ceramic capacitors are not especially suited to precision timing applications[7].

\subsection{Modulation}

Modulation was achieved using the 74HCT132 NAND Schmitt trigger. The Schmitt Trigger function helps to stabilize circuit oscillation. This method is normally used in IR remote controls as it offers a high degree of noise immunity against interfering light sources. The outputs from 555 timer and the HT12E encoder feed a dual input Schmitt trigger 74HCT132 NAND gate which does the actual phase comparison. This combination of both inputs to the NAND will give us the $38 \mathrm{KHz}$ modulated data signal with $50 \%$ duty cycle, on the output pin of the NAND. We are actually controlling the carrier frequency with our serial data-stream by turning the carrier ON/OFF for the duration of each data-bit.

The NAND output (pin \#3 Fig. 4) will only go to logic 0 when both inputs $1 \& 2$ are at logic 1 . This effectively holds the PNP drive transistor OFF until both inputs are at logic 1(Fig. 5). Since the carrier frequency is 38 $\mathrm{KHz}$, and considerably faster than our serial data, the serial data will cause the transistor to turn ON during each logic 1 data-bit.

\subsubsection{Schmitt Trigger}

The Schmitt Trigger is used here for re-shaping and filtering the signal before applying to the transistor deriver. The 74HCT132 are high-speed Silicon-gate CMOS devices and are pin compatible with low power Schottky TTL (LSTTL). The 74HCT132 contain four 2-input NAND gates which accept standard input signals [5] .They are capable of transforming slowly changing input signals into sharply defined, jitter-free output signals. The gate switches at different points for positive and negative-going signals.

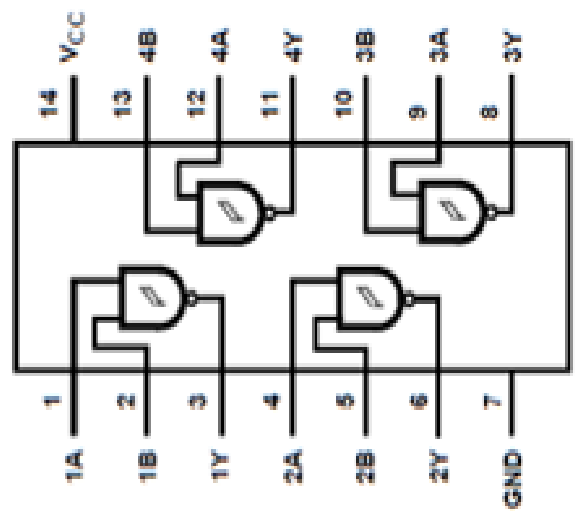

Fig. 7 Functional diagram of the 74HCT132 NAND Schmitt Trigger

\section{INFRARED EMITTER}

It is desirable the transmitteremits IR rays that can be picked up by the phototransistor or photodiodes. Since, there will be IR rays present during the day from the sunlight; the receiver will be detecting all the rays from other sources. The only way of avoiding this occurrence is by coding the IR rays from the transmitter. Consequently, the receiver should be designed to react only to those particular coded rays. The simplest way of coding is to make the signal to oscillate at a specific frequency. The receiver will then be tuned to that frequency. In this design coding is done using Holtek's HT12D decoder and HT12E encoder then transmission of IR signal at $38 \mathrm{KHz}$.

\section{INFRARED DETECTOR}

To avoid unnecessary detections a special type of phototransistor or photodiode is required and this should be immune to sunlight. The detector used in this design is a KSM-803LM optic remote control receiver module .It consists of a Pin photodiode of high speed and a preamplifier IC in the package. It is TTL and CMOS compatible. 


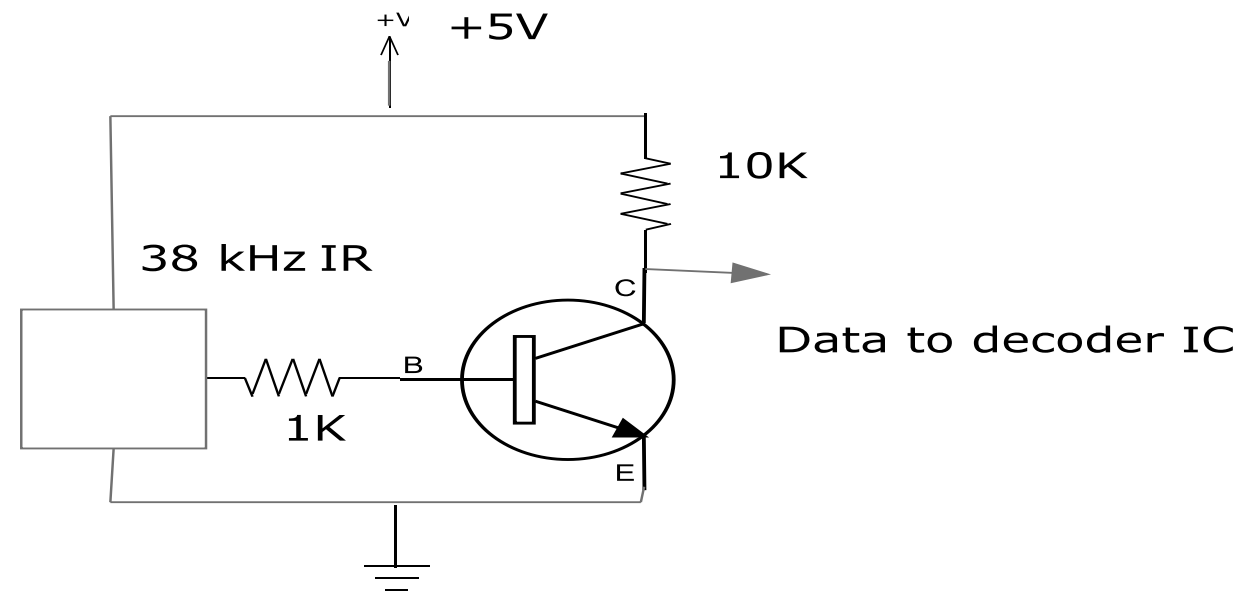

Fig.8Circuit for use with Holtek decoder ICs.

The circuit shown inFig. 8 is a simple inverter circuit, and necessary for use with the HT-12D and other decoders. The KSM803LM output has an internal pull-up resistor. This causes the output to hold logic 1 (high) on the base of the NPN transistor. The HT-12D decoder IC will go into low power or sleep mode when the data input pin is at ground conserving power in the receiver circuit until data is being received [5].The NPN transistor will complete the double inversion to retrieve the original signal which is another security feature of this circuit.

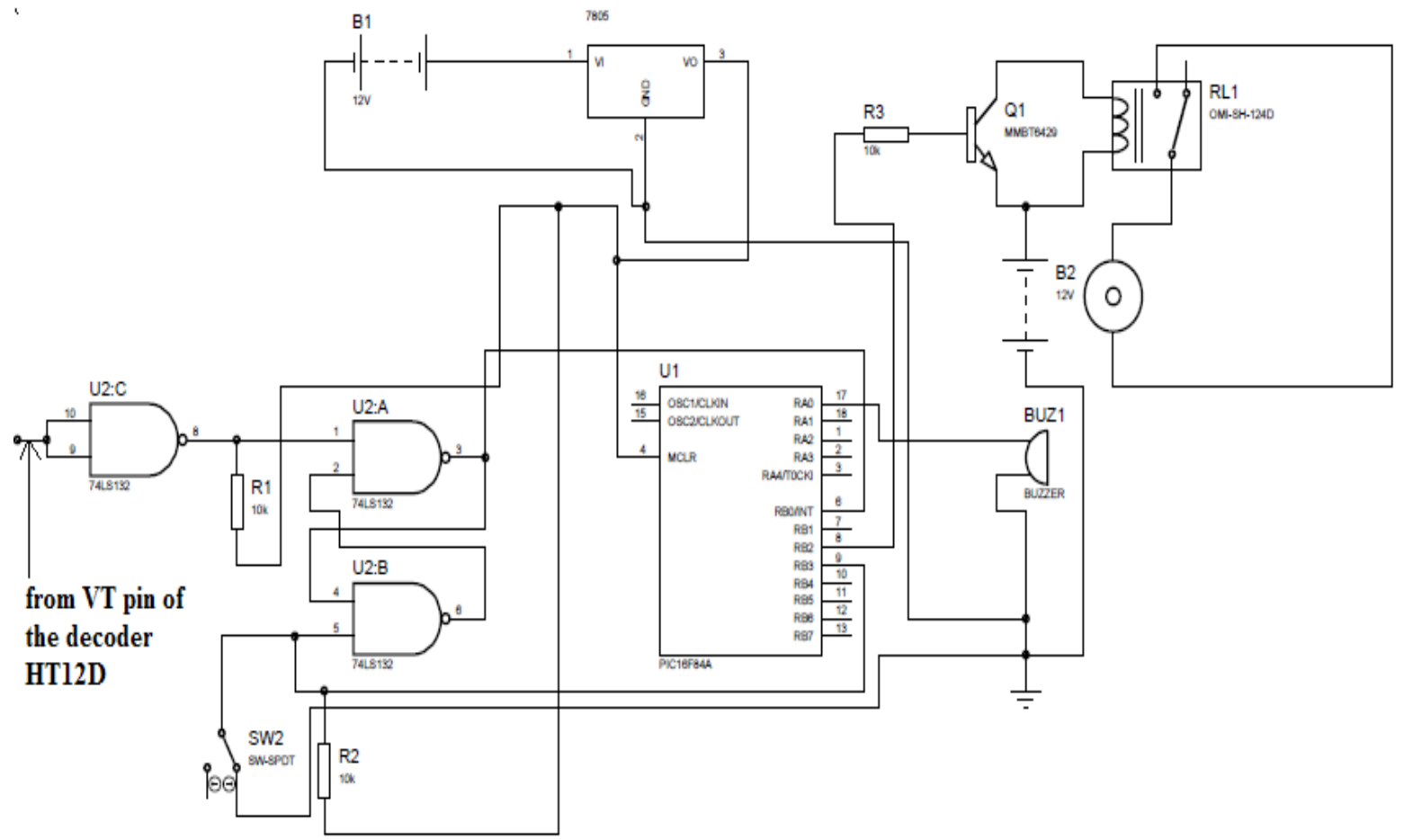

Fig. 9 Receiver Circuit

\subsection{Circuit Operation}

The Holtek HT-12E IC encodes 12-bits of information and serially transmits this data on receipt of a Transmit Enable, or a LOW signal on pin-14 /TE. Pin-17 the D_OUT pin of the HT-12E serially transmits whatever data is available on pins $10,11,12$ and 13 . Data is transmitted at a frequency selected by the external oscillator resistor. The data output pin of the encoder provides the serial data on the transmitter [5].

By using the switches attached to the data pins on the HT-12E, as shown in Fig.9, we can select the information in binary format to send to the receiver. The receiver section consists of the infrared receiver module, microprocessor and the HT-12D decoder IC. The DATA_IN pin-14 of the HT-12D reads the 12-bit binary information sent by the HT-12E and then places this data on its output pins. 
The HT-12D receives the 12-bit word and interprets the first 8-bits as address and the last 4-bits as data. Pins 1-8 of the HT-12E are the address pins. Using the address pins of the HT-12E, we can select different addresses for up to 256 receivers. The address is determined by setting pins 1-8 on the HT-12E to ground, or just leaving them open. The address selected on the HT-12E circuit must match the address selected on the HT$12 \mathrm{D}$ circuit exactly, or the information will be ignored by the receiving circuit. When the received addresses from the encoder matches the decoders, the Valid Transmission pin-17 of the HT-12D will go HIGH to indicate that a valid transmission has been received and the 4-bits of data are latched to the data output pins, 10-13. The transistor circuit shown in the schematic (Fig.8) will use the VT, or valid transmission pin to interrupt the execution of code in the microprocessor so that the relay circuit is activated so that the train stops. When the VT pin goes HIGH it turns on the 2 N2222 transistor which in turn delivers power to the LED providing a visual indication of a valid transmission reception.

\subsection{Flow Diagram:}

\section{LOGICAL FUNCTION OF THE PIC}

The sequences of events are as shown in the Fig. 10 the control shall be executed by the microprocessor chip called pic16F84A as shown in the following flow diagram Fig.9.Coding of the microprocessor was achieved using PROTON, which is a PIC Basic High Level programming language[8].

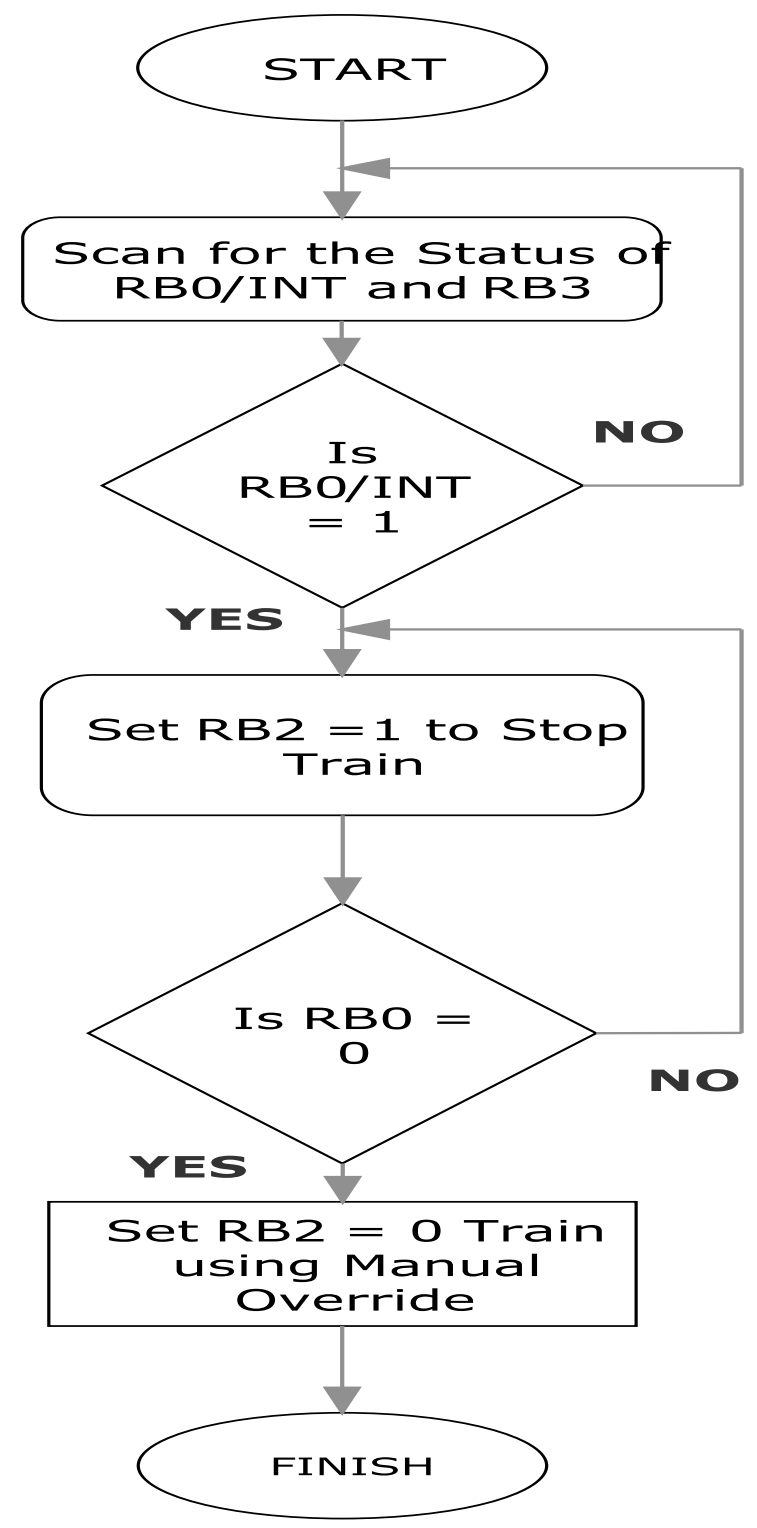

Fig.10 Flow chart for the PIC 16F84A chip operation 


\section{CONCLUSION AND RECOMMENDATIONS}

This system could be made more efficient by incorporating the Global Positioning System to give the actual geographical position of the locomotives relative to each other. This system is also ideal in that traffic density is increased and there is an accompanying improvement in the service delivery. In the case of derailment or a fault, maintenance personnel can be easily and quickly deployed to the scene using this system as the actual movement of the locomotive is being traced and monitored.

This anti-collision and detection system could also be implemented using side transceivers along the rail track to enhance the current radio communication infrastructure. In this system a train radio communication will include a plurality of land communication and train communication equipment. The implementation of this project would also require an ideal platform and infrastructure such as locomotives with electronically controlled pneumatic braking system, which makes the integration very easy.

\section{Textbooks}

\section{REFERENCES}

[1] Train accident Reconstruction and FELA and Railroad Litigation: James R Loumiet, William G. Ungbauer, and Bernard S Abrams.

[2] Calculations of braking Distance: ACM Digital Library by Daniel Banarney, David Haley and George Nikandros

[3] Advance level Mathematics $4^{\text {th }}$ Edition by C. J Tranter, CG Lambe published by Hodder and Stoughton Ltd

[4] Signal and Operational systems: Queensland Rail Brisbane Australia by David Barney Computer Society Inc. Darling Hurst Australia 201

[5] Rapid Data sheet by Holtek published: April 112000

[6] Principles of electronic circuits: Thomas R. Floyd. Edition 1997 Prentice Hall

[7] Electronic Instruments and Instrumentation. Larry D. Jones and A. Foster Chin. 1991Englewood Cliffs, NJ: Prentice Hall

[8] Modern Electronic Instrumentation and Measurement Techniques. AlbertD. Helfick and William D.Cooper. 1991 Prentice Hall

[9] Proton Programming Language Reference Manual by Brian Campbell: published 1993 by Apple. 